\title{
ON THE EXISTENCE OF COMPLETE BOOLEAN ALGEBRAS WHOSE PRINCIPAL IDEALS ARE ISOMORPHIC TO EACH OTHER
}

SEYMOUR GINSBURG

At a recent meeting of the American Mathematical Society B. Jónsson proposed the question of the existence of a complete Boolean algebra, other than the measure algebra of the unit interval modulo sets of measure zero, having the property (hereafter called property $\left.\left({ }^{*}\right)\right)$ that all principal ideals ${ }^{1}$ are isomorphic to each other. The purpose of this note is to show the existence of such Boolean algebras. The method used is new and appears applicable to other questions relating to complete Boolean algebras.

Following the terminology in [1], a nonempty subset $R$ of the partially ordered set $P$ is said to be residual if for each element $p$ in $R$, $\{x \mid x \leqq p, x \in P\}$ is a subset of $R$. A subset $Q$ of $P$ is cofinal in the subset $R$ of $P$ if for each element $r$ in $R$, there exists $q$ in $Q$ such that $q \leqq r$ in $P$. The subset $M$ of $P$ is maximal residual if $M$ is residual and if for no residual subset $N$ of $P$ is $M$ a proper cofinal subset of $N$.

As noted in [1] the family of all maximal residual subsets, ordered by set inclusion, together with a zero element, forms a complete Boolean algebra.

TheOREM. For each transfinite cardinal number $\boldsymbol{\aleph}_{\alpha}$, there exists a complete Boolean algebra, of power $2^{\aleph \alpha}$, having property $(*)$.

Proof. Let $P$ be the set of all finite sequences of ordinal numbers $<\omega_{\alpha}$, i.e., let a typical element of $P$ be $\left(x_{0}, \cdots, x_{n}\right)$ where each $x_{i}<\omega_{\alpha}$. Let the elements of $P$ be ordered as follows: $\left(x_{0}, \cdots, x_{n}\right)$ $\leqq\left(y_{0}, \cdots, y_{m}\right)$ if and only if $m \leqq n$ and $x_{i} \leqq y_{i}$ for $i \leqq m$. For each element $p$ in $P$ let $A(p)=\{x \mid x \leqq p, x \in P\}$. Let $B$ be the family of all maximal residual subsets of $P$, together with a zero element, ordered by set inclusion.

For each residual subset $X$ of $P$ let $M(X)$ be the maximal residual subset of $P$ in which $X$ is cofinal.

The power of $P$ is $\sum_{n} \aleph_{\alpha}^{n}=\boldsymbol{\aleph}_{\alpha}$. The subset $D=\left\{y_{\xi} \mid y_{\xi}=(\xi), \xi<\omega_{\alpha}\right\}$ of $P$ is such that $A\left(y_{\xi}\right) \cap A\left(y_{v}\right)$ is empty for $\xi \neq v$. For each $\xi<\omega_{\alpha}$ let $q_{\xi} \leqq y_{\xi}$ and let $Q=\left\{q_{\xi} \mid \xi<\omega_{\alpha}\right\}$. Let $Z$ be the family of all possible sets $Q$. The power of $Z$ is $2^{\aleph \alpha}$. For $Q_{1} \neq Q_{2}$ in $Z$,

Received by the editors December 12, 1956.

${ }^{3}$ By principal ideal of a Boolean algebra $B$ we shall mean an ideal which is generated by a nonzero element of $B$. 


$$
M\left(\bigcup_{q_{\xi} \in Q_{1}} A\left(q_{\xi}\right)\right) \neq M\left(\bigcup_{q_{\xi} \in Q_{2}} A\left(q_{\xi}\right)\right) .
$$

Consequently there are at least, and thus exactly, $2^{\aleph \alpha}$ elements in $B$.

For each element $N \neq 0$ in $B$, let $I(N)$ be the principal ideal generated by $N$. Clearly $I(N)$ consists of $N$ and all elements $X$ of $B$ such that $X \leqq N$. To prove that the ideal $I\left(N_{1}\right)$ is algebraically isomorphic to the ideal $I\left(N_{2}\right)$ for arbitrary $N_{1}, N_{2}, \neq 0$, it is sufficient to show that $I\left(N_{1}\right)$ is order-isomorphic ${ }^{2}$ to $I\left(N_{2}\right)$. To prove this it is sufficient to show that for arbitrary $N \neq 0$ in $B, I(N)$ is order-isomorphic to $I(A(0))$, where $A(0)=\{p \mid p \leqq(0), p \in P\}$. Note that " 0 " in "(0)" is the integer zero.

We now observe that for each maximal residual set $N$, there is a subset $E(N)=\left\{p_{\xi} \mid \xi<\omega_{\alpha}\right\}$ of $N$ such that

(1) $A\left(p_{\xi}\right) \cap A\left(p_{v}\right)$ is empty for $\xi \neq v$; and

(2) $\mathrm{U}_{\xi<\omega_{\alpha}} A\left(p_{\xi}\right)$ is a cofinal subset of $N$.

For example, suppose that $\{q\} \cup\left\{z_{\gamma} \mid \gamma\right\}$ is the set of maximal elements of $N$, with $q=\left(x_{0}, \cdots, x_{n}\right)$. One possible selection of $E(N)$ is $\left\{z_{\gamma} \mid \gamma\right\} \cup\left\{\left(x_{0}, \cdots, x_{n}, x\right) \mid x<\omega_{\alpha}\right\}$.

Let $N \neq 0$ be an arbitrary element of $B$. There exists $E(N)$ satisfying (1) and (2) above. Let $E(A(0))=\left\{x_{\xi} \mid \xi<\omega_{\alpha}\right\}$ satisfy (1) and (2) above. It is evident from the definition of $P$ that each $A\left(p_{\xi}\right)$ is orderisomorphic, under a mapping $f_{\xi}$ to $A\left(x_{\xi}\right)$. Let $R$ be any nonzero element of $I(N)$. Then $R$ is a nonempty subset of $N$. Let

$$
f(R)=M\left[\bigcup_{\xi<\omega_{\alpha}} f_{\xi}\left(A\left(p_{\xi}\right) \cap R\right)\right] .
$$

Define $f(0)=0$.

Trivially $f$ is order preserving. Suppose that $N_{1}$ and $N_{2}$ are two distinct elements of $I(N)$. Since $N_{1} \neq N_{2}$, there exists $p_{\xi}$ in $E(N)$ such that either $N_{1} \cap A\left(p_{\xi}\right)$ is not cofinal in $N_{2} \cap A\left(p_{\xi}\right)$ or vice-versa. Assume the former, an analogous argument holding if the latter. Then $f_{\xi}\left[N_{1} \cap A\left(p_{\xi}\right)\right]$ is not cofinal in $f_{\xi}\left[N_{2} \cap A\left(p_{\xi}\right)\right]$. Thus $f\left(N_{1}\right)$ is not cofinal in $f\left(N_{2}\right)$. Consequently $f$ must be one to one. Let $T$ be any maximal residual set $\leqq(0)$. Let $^{3}$

$$
R=M\left[\bigcup_{\xi<\omega} f_{\xi}^{-1}\left(A\left(x_{\xi}\right) \cap T\right)\right] .
$$

2 The partially ordered set $(S, \leqq)$ is order-isomorphic to the partially ordered set $\left(T, \leqq^{\prime}\right)$ if there exists a one to one mapping $f$, called an order-isomorphism, of $S$ onto $T$ such that $s_{1} \leqq s_{2}$ for arbitrary $s_{1}$ and $s_{2}$ in $S$ if and only if $f\left(s_{1}\right) \leqq \prime f\left(s_{2}\right)$.

${ }^{3} f^{-1}$ is the inverse of the onto function $f$. 
It is easily verified that $f(R)=T$. Thus $f$ is onto. Hence $f$ is an orderisomorphism of $I(N)$ onto $I(A(0))$. This proves the theorem.

The problem arises as to whether or not for some $\boldsymbol{\aleph}_{\delta}$, any two complete Boolean algebras, both having the same power $\geqq \boldsymbol{\aleph}_{\delta}$ and both having property $\left({ }^{*}\right)$, are isomorphic. The answer is in the negative as we now show. Let

$$
\boldsymbol{\aleph}_{\alpha}=\boldsymbol{\aleph}_{\omega_{\delta+1}} \sum_{\xi<\omega_{\delta+1}} 2^{\boldsymbol{\aleph}_{\xi}}
$$

Let $P$ and $B$ be the partially ordered set and Boolean algebra respectively for $\boldsymbol{\aleph}_{\alpha}$ as obtained in the theorem. Then the power of $B$ is $2^{\boldsymbol{N}_{\alpha}}$. Let $Q$ be the set of all zero-one sequences $\left(x_{0}, \cdots, x_{\xi} \cdots \mid \xi<\nu\right)$, $1 \leqq \nu<\omega_{\omega_{\delta}+1}$, ordered as follows: $\left(x_{0}, \cdots, x_{\xi} \cdots \mid \xi<\nu\right)$ $\leqq\left(y_{0}, \cdots, y_{\xi} \cdots \mid \xi<\mu\right)$ if and only if $\mu \leqq \nu$ and $x_{\xi}=y_{\xi}$ for $\xi<\mu$. Let $C$ be the family of all maximal residual subsets of $Q$, together with a zero element, ordered by set inclusion. The power of $Q$ is

$$
\boldsymbol{\aleph}_{\alpha}=\boldsymbol{\aleph}_{\omega_{\delta+1}} \sum_{\xi<\omega_{\delta+1}} 2^{\boldsymbol{N}_{\xi}}
$$

and the power of $C$ is $2^{\text {ka }}$. As in the theorem, one can show that $C$ has property $(*)$. Suppose that $B$ is isomorphic to $C$. Without loss of generality, we may identify $B$ with $C$. By Theorem 5 of $[1], P$ is mapped cofinally into $B-\{0\}$ and $Q$ cofinally into $C-\{0\}$. Let $q_{0}$ be any element of $Q$ and $p_{0}<q_{0}$ in $B$. The element $p_{0}$ exists since $P$ is a cofinal subset of $B-\{0\}$ and thus cofinal in $Q$. Suppose that for each $i \leqq n, p_{i}$ and $q_{i}$ are elements of $P$ and $Q$ respectively such that $p_{i}<q_{i}$. Let $q_{n+1}$ be an element of $Q$ and $p_{n+1}$ of $P$ such that $p_{n+1}<q_{n+1}$ $<p_{n} . q_{n+1}$ and $p_{n+1}$ exist due to the cofinal properties of $P$ and $Q$ in $B-\{0\}$. In this way we obtain the two simply ordered sets $\left\{p_{n} \mid n<\omega\right\}$ and $\left\{q_{n} \mid n<\omega\right\}$. Since $\omega$ is not cofinal in $\omega_{\omega_{\delta+1}}$ it readily follows that there must exist an element $q_{\omega}$ in $Q$ such that $q_{\omega}<q_{i}$ for each integer $i$. Then there exists an element $p_{\omega}$ in $P$ such that $p_{\omega}<q_{\omega}$. Then $\left\{p_{\xi} \mid \xi \leqq \omega\right\}$ is a simply ordered subset of $P$ of order type $1+\omega^{*}$. But this is impossible since the construction of $P$ demands that each simply ordered subset of $P$ be either finite or of order type $\omega^{*}$. Consequently $B$ is not isomorphic to $C$.

\section{BIBLIOGRAPHY}

1. S. Ginsburg, A class of everywhere branching sets, Duke Math. J. vol. 20 (1953) pp. 521-526.

University of Southern CALIFornia,

NORTHROP AIRCRAFT INC. AND

National Cash Register Company 